\title{
Jovens e Adultos Construindo e Interpretando Gráficos
}

\section{Youth and Adults Building and Interpreting Graphs}

\author{
Izauriana Borges Lima* \\ Ana Coêlho Vieira Selva**
}

\begin{abstract}
Resumo
O objetivo deste estudo foi analisar 30 estudantes da EJA, em diferentes níveis de escolarização, resolvendo atividades de construção e interpretação de gráficos. Os resultados não mostraram diferenças significativas no desempenho dos estudantes nas atividades de interpretação em função da escolaridade. Na construção dos gráficos várias dificuldades foram observadas. A maioria dos gráficos construídos não apresentou informações necessárias para a compreensão do mesmo (título, nomeação dos eixos, descrição das variáveis). Dificuldade com a escala foi um dos aspectos mais evidentes. Observamos que os desempenhos dos alunos que conseguiram realizar com sucesso as atividades de interpretação não garantiram a construção adequada de um gráfico. Este dado sugere que há pouca relação entre tais atividades e que interpretar parece ter sido mais fácil que construir. Os dados sugerem que há necessidade de maior estímulo à construção de gráficos e que o professor articule as atividades de interpretação com as de construção.
\end{abstract}

Palavras-chave: Educação de Jovens e Adultos. Interpretação e Construção de Gráficos. Escolarização.

\footnotetext{
* Mestre em Educação Matemática e Tecnológica pela Universidade Federal de Pernambuco (UFPE), Recife, PE, Brasil. Professora das séries iniciais do Ensino Fundamental da Prefeitura Municipal de Jaboatão dos Guararapes, PE, Brasil. Endereço para correspondência: Rua Nova Canaã, n. 210, Apt ${ }^{\circ}$ 104, Jordão Baixo. Recife, PE, Brasil. E-mail: dainha.borges@ hotmail.com.

** Doutora em Psicologia Cognitiva pela Universidade Federal de Pernambuco (UFPE), Recife, PE, Brasil. Professora Adjunta da Universidade Federal de Pernambuco (UFPE). Endereço para correspondência: Av. Acadêmico Hélio Ramos, s/n, Centro de Educação-DPOE. Cidade Universitária, Recife, PE, Brasil. E-mail: anaselva@globo.com.
} 


\begin{abstract}
The aim of this study was to analyze adult education students at different levels of education, solving activities of construction and interpretation of graphs. The results showed no significant differences in student performance in activities of interpretation depending on the level of schooling. In the construction of several graphics problems were observed. Most of the plotting did not provide the information necessary to understand it. Difficulty with the scale was one of the most evident among the students. We observed that the performance of students who have achieved success with the activities of interpretation failed to ensure proper construction of a chart. This suggests that there is little relationship between such activities and that graphs interpretation was easier than construction. The data suggest that there is a need to encourage the construction of charts and for teachers to integrate activities of interpretation with the construction of graphs.
\end{abstract}

Keywords: Youth and Adults. Interpret and Construct Graphs. Schooling.

\title{
1 Introdução
}

O objetivo deste trabalho foi investigar o desempenho de estudantes da Educação de Jovens e Adultos (EJA) em atividades de construção e interpretação de gráficos. Especificamente, investigamos como a escolarização influenciou o desempenho dos jovens e adultos ao interpretarem e construírem gráficos. Assim, participaram da pesquisa estudantes da EJA no último ano dos anos iniciais, no último ano dos anos finais do Ensino Fundamental e no último ano do Ensino Médio.

Para compreender o universo dessa modalidade de ensino e suas especificidades, procuramos autores que se dedicaram em refletir o significado de ser sujeito excluído do sistema regular de ensino e o tratamento que vem sendo dado a esse público que inicia ou retoma o processo de aprendizagem escolar tardiamente. Serviu, ainda, como base teórica, documentos de referência legal que orientam o currículo da EJA e pesquisas desenvolvidas acerca do conhecimento matemático relacionadas ao eixo Tratamento da Informação.

Para propiciar uma melhor compreensão do desenvolvimento deste estudo, nos tópicos seguintes discutimos alguns elementos acerca das peculiaridades da Educação de Jovens e Adultos, a importância do Tratamento da Informação na Educação Matemática e na EJA. Posteriormente, apresentamos a metodologia utilizada, os resultados obtidos e as considerações finais a respeito desta pesquisa. 


\section{Educação de Jovens e Adultos: uma modalidade de ensino}

Para discutir sobre Educação de Jovens e Adultos é imprescindível atentarmos ao fato de que, para entender o grupo que constitui essa modalidade de ensino, não basta apenas compreendê-lo por sua característica etária, mas, também, por suas especificidades socioculturais que os definem enquanto sujeitos de conhecimento e aprendizagem. Oliveira (1999, p. 60) destaca três campos que contribuem para compreensão sobre como jovens e adultos pensam e aprendem, e para definir a posição social que ocupam: "a condição de —nãocriança, a condição de excluídos da escola e a condição de membros de determinados grupos sociais".

A escola para jovens e adultos possui uma estrutura organizacional e hierárquica acerca do conhecimento que, muitas vezes, é estranha aos adultos e jovens que a ela se destinam. As normas e os currículos, geralmente, têm sido os mesmos utilizados no trabalho realizado com crianças, tornando a escola um lugar inadequado ao atendimento das especificidades de alunos de faixas etárias mais avançadas, que não concluíram os estudos na idade própria.

A Educação Matemática de Jovens e Adultos deve ser entendida enquanto uma ação pedagógica destinada a estudantes cujas causas e efeitos da interrupção, ou o não acesso ao ensino regular em idade própria, estão inseridos num amplo contexto de exclusão social e cultural. Ao mesmo tempo, entendemos que sempre é tempo de aprendizagens.

Algumas pesquisas em Educação foram realizadas com o objetivo de investigar, especificamente, conhecimentos matemáticos que jovens e adultos possuem, ainda que não tenham tido acesso ao ensino formal, ou que tiveram passagens mal sucedidas na escola. Tais investigações (SILVA, 2006; GOMES, 2007; entre outras) têm contribuído para a valorização da Matemática construída a partir de experiências cotidianas que muitos jovens e adultos desenvolveram, bem como na reflexão de que uma EJA significativa, para os que agora iniciam ou reiniciam o processo de sistematização formal de ensino, é aquela capaz de valorizar o conhecimento que os alunos trazem para a escola como ponto de partida para novas aprendizagens.

Considerando que o conhecimento matemático vem sendo desenvolvido em situações cotidianas, ainda que a escola tenha um papel fundamental de sistematização e ampliação desses conhecimentos, devemos compreender, especialmente no que se refere à interpretação e construção de gráficos, foco da presente pesquisa, a importância desse conhecimento e de seus processos 
de aprendizagem. Para isto, no próximo tópico, iniciamos uma discussão sobre o bloco Tratamento da Informação e, mais especificamente, sobre alguns aspectos relativos à representação gráfica.

\section{Tratamento da Informação}

De acordo com Lopes (2009, p. 3)

a presença constante da Estatística no mundo atual tornoua uma realidade dos cidadãos, levando à necessidade de ensinar Estatística a um número de pessoas cada vez maior. Conseqüentemente, nos últimos 50 anos a maioria dos países introduziu, nos seus programas de Matemática, conteúdos de Estatística, na forma de uma unidade curricular.

No que concerne à realidade da educação no Brasil, os conceitos de Estatística foram incluídos no bloco de conteúdos matemáticos denominado Tratamento da Informação, que integra as noções de estatística, probabilidade e combinatória.

Ressaltamos que, no Ensino Fundamental e Médio, a Estatística não é trabalhada como um componente curricular específico, mas apresenta seu conteúdo distribuído em outras disciplinas, como a Matemática, sendo trabalhado no eixo Tratamento da Informação, geralmente representado pela compreensão de gráficos e tabelas.

A incorporação das noções de análise de dados estatísticos no currículo da Educação Básica se fundamenta na importância alcançada pelo tratamento da informação nos dias de hoje. Convivemos com uma circulação elevada de informações e formas particulares de apresentação dos dados, que exigem o desenvolvimento do raciocínio estatístico para solucionar, compreender e atuar frente às diversas situações representadas. Raciocínio estatístico é definido por Garfield (2002) como a maneira como as pessoas pensam com as ideias estatísticas e como dão sentido à informação estatística. Isto envolve fazer interpretações baseadas em conjuntos de dados, representações gráficas e resumos estatísticos.

O desenvolvimento do bloco Tratamento da Informação justifica-se, ainda, segundo os Parâmetros Curriculares Nacionais (BRASIL, 1998, p. 134), por ser um campo que abarca uma ampla variedade de conteúdos matemáticos e pode 
favorecer o aprofundamento, a ampliação e a aplicação de conceitos e procedimentos como porcentagem, razão, proporção, ângulo, cálculos etc. Esse estudo também favorece o desenvolvimento de certas atitudes, como posicionar-se criticamente, fazer previsões e tomar decisões ante as informações veiculadas pela mídia, livros e outras fontes.

Discutindo a questão da relação entre a Matemática e a Estatística, Lopes (2004) e Lopes e Carvalho (2005) consideram que o fato da Estatística ser ensinada dentro de outras áreas de conhecimento, sobretudo na Matemática, faz com que seja trabalhada, frequentemente, com ênfase no uso de cálculos através da aplicação de fórmulas e em procedimentos mecânicos para a elaboração de representações de dados, sem priorizar uma atividade reflexiva acerca do impacto dessas representações. Acrescentam, ainda que, essa forma de lidar com as duas disciplinas resulta no julgamento de que os pensamentos estatístico e matemático são semelhantes.

Apesar da aproximação, essas duas áreas de conhecimento possuem características peculiares que as diferenciam entre si. Entretanto, a Estatística tem sido trabalhada no ensino da Matemática, o que exige uma análise cautelosa do trabalho que vem sendo realizado com os conteúdos estatísticos em especial nas aulas de Matemática na Educação Básica.

Os impactos do ensino de conteúdos inerentes a Estatística como parte do ensino da Matemática remete-nos a refletir, também, a concepção em torno da ideia de literacia estatística. Lopes (2004, p. 187) define literacia estatística como a habilidade dos sujeitos em compreenderem e interpretarem dados estatísticos em textos jornalísticos, notícias e informações de diferentes naturezas.

Assim, diante de dados de natureza quantitativa não é suficiente possuir somente habilidades matemáticas para entender o fenômeno que está sendo representado, é extremamente relevante o desenvolvimento de habilidades estatísticas necessárias à compreensão das informações. De acordo com Lopes (2004, p. 188) a aquisição de habilidades relativas à literacia estatística "requer o desenvolvimento do pensamento estatístico o qual permite que a pessoa seja capaz de utilizar idéias estatísticas e atribuir um significado à informação estatística". Para tanto, os sujeitos terão que ser capazes de interpretar a realidade a partir de um conjunto de dados ou de sua representação. Considera, ainda, que pensar estatisticamente consiste em somar as ideias subjacentes aos dados disponíveis e incertezas, realizar inferências e, ao mesmo tempo, conhecer 
determinados conceitos inerentes à Estatística, "como a distribuição de frequiências, medidas de posição e dispersão, incerteza, acaso e amostra".

Lopes (2004) defende que a Educação Estatística tenha como eixo central a análise exploratória dos dados. De acordo com a autora, a incorporação do ensino da Estatística nas aulas de Matemática parece exigir que os conhecimentos estatísticos sejam abordados na perspectiva da análise de dados, a partir da definição de uma questão ou problemática significativa para os estudantes. Se os conceitos estatísticos forem trabalhados de forma desvinculada de uma problemática relacionada à realidade social na qual o sujeito se insere, a atividade proposta fará pouco ou nenhum sentido. Propor a realização da coleta de dados ou a leitura e construção de representações gráficas dissociadas do contexto, ou das situações próximas aos alunos, não garante possibilidades de uma análise crítica da realidade. Assim, a autora recomenda que a escola oportunize possibilidades reais, que façam sentido para os alunos, de realizarem tratamento de dados, em todas as suas etapas, favorecendo o desenvolvimento do raciocínio estatístico.

É importante ressaltar que a representação dos dados em gráficos e/ou tabelas, considerando a relevância dessa etapa no processo de tratamento de dados, isto é, em conjunto com os aspectos relativos ao planejamento das investigações e a realização de inferências, legitima a importância de estudos mais aprofundados na análise do desempenho que os sujeitos apresentam em relação a essa área de conhecimento. Bem como, contribui na busca por novos caminhos que possam contribuir cada vez mais com os processos de ensino e aprendizagem.

\section{Tratamento da Informação na Educação de jovens e adultos}

Nos últimos anos, observamos um aumento no número de estudos que investigaram a compreensão de gráficos e tabelas envolvendo crianças (SELVA, 2003; GUIMARÃES, 2002; GUIMARÃES; GITIRANA; ROAZZI, 2001, entre outros). Em relação a jovens e adultos, ainda são poucos os estudos na área de Matemática, especialmente, sobre o conhecimento estatístico (GITIRANA; GUERRA; SELVA, 2005).

Apresentamos, a seguir, algumas dessas pesquisas para melhor elucidar as questões relacionadas ao desempenho de crianças e adultos escolarizados nas atividades com os gráficos e/ou tabelas. A partir da análise dos resultados encontrados nesses estudos serão levantadas algumas reflexões relacionadas 
ao tratamento de dados estatísticos no ensino de jovens e adultos, objetivo deste estudo.

O principal objetivo do trabalho realizado por Guimarães, Gitirana e Roazzi (2001) foi investigar a compreensão da interpretação de gráficos de barras, a construção dos gráficos de barras a partir de dados apresentados em tabelas e a relação entre interpretação e construção. A pesquisa foi realizada com 107 alunos, de quatro turmas da $3^{\mathrm{a}}$ série $/ 4^{\circ}$ ano do Ensino Fundamental, em uma escola particular no estado de Pernambuco.

Todos os alunos foram solicitados pelo experimentador a resolverem cinco atividades: duas de interpretação de gráficos com dados nominais, uma de interpretação de gráficos com dados ordinais e duas de construção de gráficos.

Os resultados da pesquisa revelaram que os alunos apresentaram facilidade em localizar pontos extremos independente do tipo de variável ser nominal ou ordinal, entretanto, quando a leitura exigia a compreensão variacional, os sujeitos encontraram dificuldades tanto para os dados nominais, quanto ordinais.

Foram observadas dificuldades com as escalas, sobretudo, quando os valores solicitados estavam implícitos, isto é, quando os valores precisavam ser inferidos a partir da escala. Os autores acreditam que essas dificuldades residem na compreensão dos valores contínuos presentes na escala, em que é necessário que os alunos estabeleçam proporcionalidade entre os pontos explicitados na escala adotada.

No que se refere às atividades de construção de gráficos, os autores chamaram atenção ao fato de que um percentual pequeno dos sujeitos da pesquisa realizou as atividades de construção, quando comparadas às atividades de interpretação. Para os autores esses resultados sugerem que interpretar gráficos pode ser mais fácil do que construir. Uma indagação que podemos fazer é: os resultados observados com crianças, por Guimarães, Gitirana e Roazzi (2001), seriam os mesmos quando se consideram os jovens e adultos? Esta é uma questão central no presente estudo.

Um outro estudo, envolvendo crianças, foi desenvolvido por Selva (2003). Ela realizou uma investigação com crianças de 6 a 8 anos na resolução de problemas aditivos, usando gráficos de barras como suporte representacional, bem como analisou as dificuldades envolvidas na atividade de interpretação e construção desse tipo de representação. Os resultados encontrados mostraram que o desenvolvimento de uma sequência de ensino, envolvendo gráficos e manipulativos, ajudou as crianças na reflexão de aspectos formais do gráfico e que as dificuldades observadas na construção e interpretação de gráficos foram 
superadas com o auxílio de outros conhecimentos matemáticos e atividades já familiares, como as utilizadas no referido estudo.

Assim, considerando que conhecimentos anteriores puderam potencializar o desempenho das crianças na construção e interpretação de gráficos, como observado no estudo de Selva (2003), é interessante investigar o papel da escolarização na compreensão e construção de gráficos com estudantes jovens e adultos. Público, este, que já possui, além da experiência de vida, conhecimentos adquiridos na própria escola ao longo das séries da EJA. Esse é um dos objetivos do presente estudo.

Guimarães (2002) realizou um estudo com crianças entre nove e dez anos de idade da $3^{\mathrm{a}}$ série $/ 4^{\circ}$ ano do Ensino Fundamental, resolvendo atividades com gráficos. Nas atividades de interpretação de gráficos de barras, a análise dos resultados indicou que as crianças apresentaram dificuldades nas questões de combinação e, principalmente, de comparação. Dificuldades na resolução de problemas de comparação também foram observadas no estudo de Selva (2003), que verificou bons desempenhos nas questões de combinação. No presente estudo, também verificamos o desempenho em problemas de comparação e de combinação.

Pagan et al. (2008) realizaram um estudo diagnóstico com o objetivo de investigar e comparar as dificuldades que alunos, em diferentes níveis de escolaridade, apresentariam ao ler e interpretar gráficos e tabelas. Participaram do estudo 399 alunos da rede pública de ensino de São Paulo, sendo 159 alunos da $5^{\mathrm{a}}$ série $/ 6^{\circ}$ ano, 80 da $8^{\mathrm{a}}$ série $/ 9^{\circ}$ ano do Ensino Fundamental e 160 alunos do $2^{\circ}$ ano do Ensino Médio. Uma das questões levantadas pela pesquisa investigava se o grau de escolaridade influenciaria a capacidade dos alunos na leitura, compreensão e análise dos dados contidos em tabelas e gráficos. A análise dos resultados encontrados indicou que houve uma melhora significativa no desempenho total dos alunos, tanto na leitura de dados pontuais quanto globais contidos em gráficos e tabelas de acordo com o nível de escolarização.

O estudo de Gitirana, Guerra e Selva (2005) teve como objetivo investigar a compreensão de professoras sobre a construção e interpretação de gráficos. Oito professoras da Rede Pública de Ensino do Recife foram entrevistadas e resolveram atividades de construção e interpretação de gráficos de barras com variáveis nominais. Os resultados indicaram que as professoras não apresentaram dificuldades nas atividades de interpretação. Entretanto, nas atividades de construção, em que foram fornecidos dados retirados da mídia impressa, as professoras demonstraram dificuldades em representar informações necessárias à compreensão do gráfico para um possível leitor. Assim, observaramse a falta de legendas, títulos e/ou nomeação dos eixos, dificuldades em relação 
ao cálculo da área dos gráficos de setores e em relação à construção da escala nos gráficos de barras. Estes dados, como também os obtidos no estudo de Guimarães, Gitirana e Roazzi (2001), indicaram uma relação independente entre as atividades de interpretação e construção de gráficos.

De modo geral, os estudos anteriormente citados contribuíram para a compreensão de questões relativas ao conhecimento matemático e estatístico inerentes ao Tratamento de Informações, principalmente em atividades de interpretação de gráficos e/ou tabelas. Entretanto, como já mencionado, a maioria dessas pesquisas foi realizada com alunos matriculados no ensino regular, sobretudo crianças, o que nos leva a pensar o quanto investigações na EJA se fazem necessárias para melhor entendermos como os estudantes dessa modalidade de ensino, que apresentam características tão peculiares, lidam com questões relacionadas ao tratamento de dados, e o quanto a escolarização tem ou não influenciado no conhecimento de noções estatísticas. Esses são objetivos do presente trabalho, que analisou o desempenho de estudantes da EJA, com diferentes níveis de escolaridade, na resolução de atividades de construção e interpretação de gráficos.

\section{Metodologia}

Este estudo se constituiu em uma pesquisa qualitativa, de caráter exploratório, cujo objetivo foi analisar o desempenho de alunos da EJA ao resolverem atividades de construção e interpretação de gráficos, em especial observando o efeito da escolarização.

Participaram desta pesquisa 30 estudantes matriculados na EJA, em escolas públicas do Recife. Os estudantes diferiam em relação ao nível de escolarização, sendo três grupos compostos por dez estudantes cada: o $G 1$ foi formado por estudantes do último ano da primeira etapa do Ensino Fundamental, o $G 2$ por estudantes do último ano da segunda etapa do Ensino Fundamental e o G3 por estudantes do Ensino Médio.

A coleta dos dados para este estudo consistiu na aplicação de um teste, envolvendo cinco atividades: três de interpretação (Gráfico Calorias, Gráfico Medalhas e Gráfico Cinema) e duas de construção (C-1 e C-2). As questões de interpretação envolveram duas atividades com gráficos de barras e uma com gráfico de linhas. As atividades com gráficos de barras consistiram em uma atividade de barras com categorias (Gráfico Calorias) e uma de barras contendo série de tempo (Gráfico Medalhas). Em relação às questões propostas, em cada gráfico os estudantes responderam a seis questões, sendo uma de análise geral do gráfico, uma de leitura pontual, duas de comparação (variação acréscimo e 
decréscimo), uma de combinação e uma de igualização. As atividades de construção consistiram na solicitação de construção de gráfico a partir de informações fornecidas. Não foi explicitado, ao estudante, o tipo de gráfico a ser construído, ficando essa escolha a critério dele.

Os gráficos usados para as questões de interpretação foram retirados de livros didáticos para o Ensino Fundamental aprovados pelo PNLD ${ }^{1}$, referentes a 2004 e 2005. Já as atividades de construção foram elaboradas aproveitandose informações de um gráfico selecionado em um livro didático (C-1, em que era apresentada a quantidade de CDs vendidos no Brasil entre 2000 e 2005) e de uma revista de grande circulação nacional (C-2, em que era apresentada a quantidade de semanas em que algumas obras do escritor Paulo Coelho estiveram em primeiro lugar).

\subsection{Atividades de interpretação de gráficos}

Observe o gráfico abaixo e responda as seguintes questões:

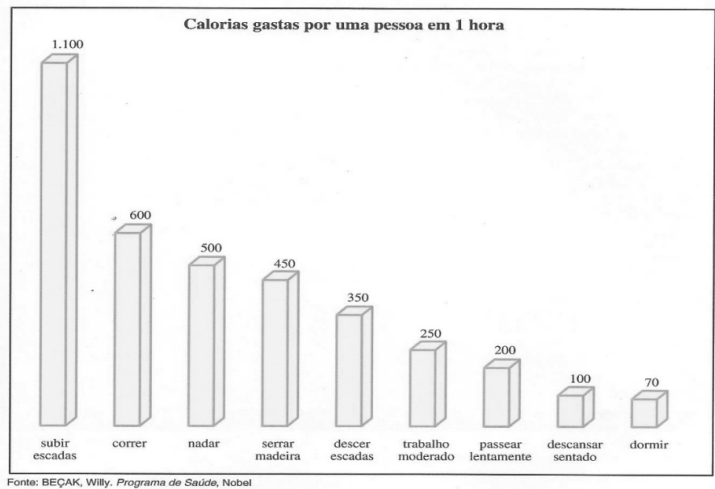

$\left.1^{\circ}\right)$ Você gostaria de fazer alguns comentários sobre o gráfico acima?

$2^{\circ}$ ) Qual é a atividade em que se gasta a maior quantidade de calorias?

$3^{\circ}$ ) Quantas calorias são gastas serrando madeira a mais do que trabalhando moderadamente?

$4^{\circ}$ ) Quantas calorias são gastas no total se uma pessoa sobe escadas durante 1 hora e desce escadas durante 1 hora?

$5^{\circ}$ ) Quantas calorias são gastas dormindo a menos do que descansando sentado?

$\left.6^{\circ}\right)$ Quantas calorias uma pessoa que nadou durante 1 hora ainda precisa gastar para ter gasto a mesma quantidade de calorias de alguém que correu durante 1 hora?

\section{Quadro 1 - Gráfico Calorias}

${ }^{1}$ Programa Nacional do Livro Didático (PNLD). O PNLD é o mais antigo dos programas do Ministério da Educação voltados à distribuição de obras didáticas aos estudantes da rede pública de ensino brasileiro. 
Observe o gráfico abaixo e responda as seguintes questões:

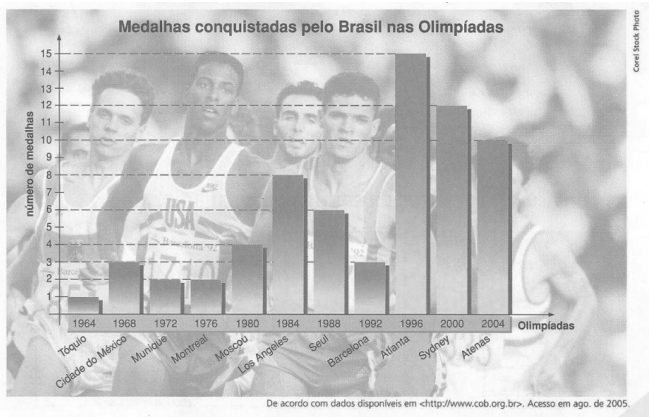

$1^{\circ}$ ) Você gostaria de fazer alguns comentários sobre o gráfico acima?

$2^{\circ}$ ) Qual foi o ano em que o Brasil conquistou o menor número de medalhas olímpicas? $3^{\circ}$ ) Quantas medalhas foram conquistadas em 1996 a mais do que em 1992?

$4^{\circ}$ ) Quantas medalhas o Brasil recebeu juntando-se as conquistadas nas Olimpíadas de 2000 e 2004 ?

$5^{\circ}$ ) Quantas medalhas foram conquistadas em 2000 a menos do que em $1996 ?$

$6^{\circ}$ ) Quantas medalhas olímpicas o Brasil precisava ainda ter conquistado em 2004 para ficar com a mesma quantidade de medalhas conquistadas em 1996 ?

\section{Quadro 2-Gráfico Medalhas}

Observe o gráfico abaixo e responda as seguintes questões:

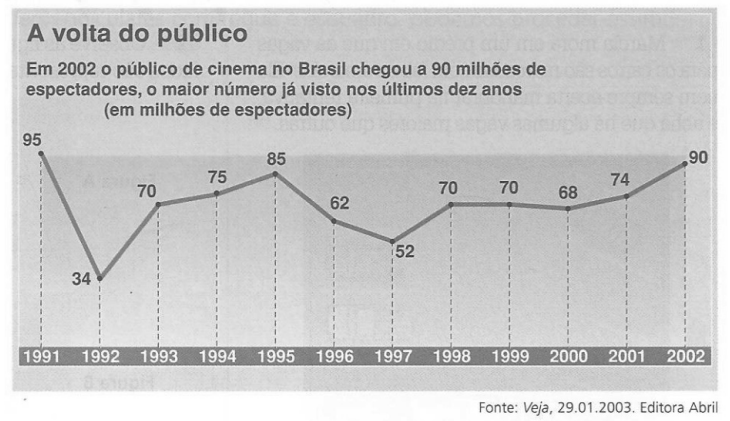

$\left.1^{\circ}\right)$ Você gostaria de fazer alguns comentários sobre o gráfico acima?

$\left.2^{\circ}\right)$ Em que ano foi registrado o maior número de espectadores nos cinemas brasileiros? $3^{\circ}$ ) Quantos espectadores foram aos cinemas brasileiros em 2002 a mais do que em 2000?

$4^{\circ}$ ) Quantos espectadores ainda precisariam ter ido ao cinema em 2001 para atingir o mesmo número de espectadores de 2002 ?

$5^{\circ}$ ) Quantos espectadores foram aos cinemas brasileiros em 1993 a menos do que em 1995 ?

$6^{\circ}$ ) Qual é o total de espectadores que foram aos cinemas brasileiros em 1999 e 2000? 


\subsection{Atividades de construção de gráficos}

De acordo com a Revista Exame, a quantidade de CD's vendidos no Brasil entre os anos de 2000 e 2005, apresentou os seguintes números:

$2000-93$ (em milhões)

$2001-70$ (em milhões)

2002- 72 (em milhões)

$2003-52$ (em milhões)

$2004-59$ (em milhões)

2005 - 46 (em milhões)

Construa um gráfico considerando as informações apresentadas acima

Quadro 4 - Atividade de construção (C-1)

A Revista Veja publicou em 2008 o resultado de uma pesquisa sobre o número de semanas em que alguns livros do escritor Paulo Coelho estiveram em primeiro lugar.

Os dados foram os seguintes:

O Alquimista - 24 semanas

Brida - 12 semanas

Na margem do rio Piedra eu sentei e chorei - 7 semanas

O Demônio e a $\mathrm{Srt}^{\mathrm{a}}$ Prym - 3 semanas

Onze minutos - 22 semanas

O Zahir - 2 semanas

Construa um gráfico considerando as informações apresentadas acima.

Quadro 5 - Atividade de construção (C-2)

\section{Resultados}

\subsection{Como jovens e adultos interpretam gráficos?}

Na Tabela 1, abaixo, apresentamos o percentual de acerto nas atividades de interpretação por tipo de gráfico e nível de escolarização dos estudantes da EJA (G1, G2 e G3). 
Tabela 1 - Percentual geral de acerto dos grupos por tipo de gráfico

\begin{tabular}{c|c|c|c|c}
\hline \multirow{2}{*}{ Grupos } & \multicolumn{4}{|c}{ Gráficos } \\
\cline { 2 - 5 } & Gráfico Calorias & Gráfico Medalhas & Gráfico Cinema & Total \\
\hline G1 & 46 & 82 & 60 & 62,6 \\
\hline G2 & 66 & 66 & 72 & 68 \\
\hline G3 & 70 & 84 & 86 & 80 \\
\hline Total & 60,6 & 77,3 & 72,6 & \\
\hline
\end{tabular}

Observando a Tabela 1, constatamos avanço no desempenho dos estudantes com a escolarização, principalmente do grupo G2 para o grupo G3. O G1 apresentou 62,6\% de acertos, G2, 68\% e G3, 80\%. Considerando os resultados acima, foi realizada uma análise de variância, tendo como variável independente o Grupo (escolarização) e variável dependente, o desempenho obtido. Os resultados não indicaram efeitos significativos da escolarização $(\mathrm{F}=0,507, \mathrm{p}=0,608)$. Esse resultado contrasta com o que foi verificado na pesquisa de Pagan et al (2008) com estudantes do ensino regular do Fundamental ao Médio, em que o desempenho em atividades com gráficos foi influenciado significativamente pelo nível de escolaridade.

Comparando o desempenho geral dos estudantes da EJA, observamos que foi no Gráfico Calorias o desempenho mais baixo $(60,6 \%)$. O melhor desempenho foi na interpretação do Gráfico Medalhas (77,3\%), seguido do Gráfico Cinema (72,6\%). Comparando o desempenho dos estudantes, verificamos diferença significativa apenas entre os gráficos de barras (Gráfico Calorias e o Gráfico Medalhas) $(\mathrm{Z}=2,330, \mathrm{p}=0,020)$. Esse resultado é interessante, pois sugere que o fato do gráfico ser de linhas ou de barras não define o grau de dificuldade do mesmo.

Os resultados indicaram que as diferenças observadas entre os estudantes não estavam relacionadas ao tipo de representação (gráfico de linhas e de barras), mas a outros aspectos constituintes dessas representações como o tipo de questão, a apresentação de certos elementos (o texto presente no Gráfico Cinema foi um fator que desencadeou o mesmo erro cometido pelos participantes na questão de leitura pontual, por exemplo), o tema abordado e, consequentemente, a mobilização de conhecimentos anteriores frente aos temas (como foi observado, sobretudo, no Gráfico Calorias).

Esse é um aspecto importante quando analisamos o trabalho, muito comum em sala de aula, de conteúdos organizados em módulos sequenciais (por exemplo, primeiro gráfico de barras, depois de linhas e, depois, de setores), sugerindo a importância de proporcionar um trabalho simultâneo com diferentes 
tipos de representação. Ainley (2000) discute esse aspecto de que não se pode organizar o trabalho com gráficos no currículo de forma segmentada, partindose da crença de que a dificuldade seria entendida como inerente ao próprio gráfico, o que, portanto, justificaria o ensino de gráficos prosseguindo no uso de formas cada vez mais complexas. Essa autora salienta a importância da análise dos diferentes aspectos envolvidos nos gráficos (escala, dados representados etc.) e não apenas o tipo de gráfico, para se organizar o desenvolvimento do trabalho escolar.

Analisando o desempenho apresentado pelos grupos em relação aos tipos de questões propostas nas atividades de interpretação dos gráficos (leitura pontual, comparação, combinação e igualização), podemos observar, no Gráfico 1 que, em geral, há um avanço no desempenho com a escolaridade, ainda que esta diferença não tenha sido significativa.

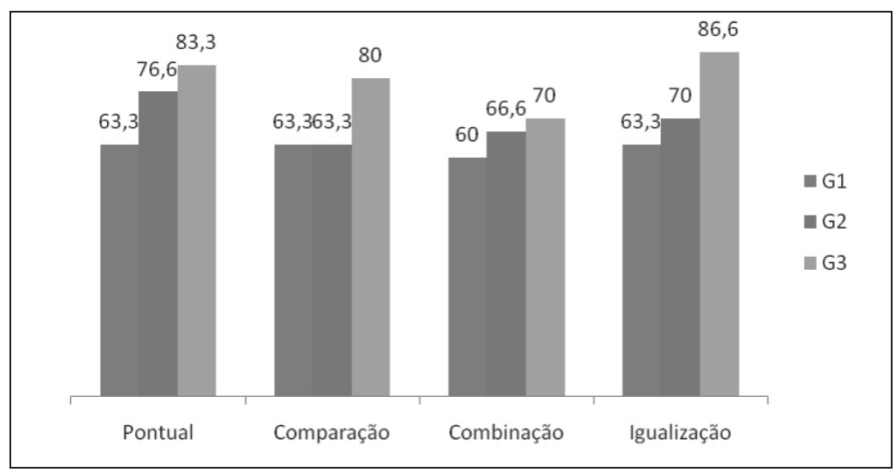

Gráfico 1 - Percentual de acerto na atividade de interpretação por tipo de questão

Considerando o desempenho dos estudantes dos anos iniciais (G1), os resultados mostram desempenhos semelhantes nos diferentes tipos de problemas das estruturas aditivas, mais baixos do que dos estudantes dos outros anos de escolaridade. Problemas de combinação e, principalmente, de comparação a partir de gráficos foram mais difíceis para os alunos dos anos finais do que questões de leitura pontual e problemas de igualização. No Ensino Médio, os resultados mais baixos aparecem nos problemas de combinação. Esse tipo de problema, quando proposto a partir de gráficos, parece trazer mais dificuldades, tal como verificado no estudo de Guimarães (2002). Observamos, ainda, que os estudantes do Ensino Médio (G3) não apresentaram muitas dificuldades nas questões de comparação.

Nos problemas de combinação, apesar de a literatura indicar que esses 
tipos de problemas são facilmente resolvidos desde os anos iniciais do Ensino Fundamental, observamos, no Gráfico Calorias, o pior desempenho. O grupo G1 apresentou apenas 30\% de acerto, enquanto os grupos G2 e G3 obtiveram 50\% de acerto. Esses dados nos chamam atenção, pois de acordo com Nunes et al. (2002) desde muito pequenos já possuímos a capacidade de coordenar o esquema de juntar com a contagem, conseguindo solucionar uma variedade de situaçõesproblema envolvendo as relações parte-todo. Entretanto, os resultados deste estudo confirmam o estudo de Guimarães (2002), que encontrou dificuldades por parte de crianças de $3^{\mathrm{a}}$ série $/ 4^{\circ}$ ano em resolver problemas de combinação. Diante desses resultados é importante que o professor trabalhe com problemas desde cedo, a partir de diferentes formas de representação, aproveitando os conhecimentos prévios de forma a dar sentido às novas informações (VERGNAUD, 1986).

Em relação à leitura pontual, nossos dados mostraram uma diferença em relação a estudos anteriores (GUIMARÃES; GITIRANA; ROAZZI, 2001; PAGAN et al., 2008; GUIMARÃES, 2002) que verificaram que esse tipo de questão é resolvida com sucesso desde cedo. Observamos, em especial no Gráfico Cinema, um percentual considerável de estudantes que responderam incorretamente a pergunta sobre o ponto máximo. Entretanto, um aspecto que pode ter contribuído para tais resultados foi a presença do comentário que acompanhava o gráfico: "Em 2002 o público de cinema chegou a 90 milhões de espectadores, o maior número já visto nos últimos dez anos", criando certa confusão para os estudantes, já que no plano cartesiano o valor mais alto correspondia ao ano de 1991.

\subsection{Como jovens e adultos constroem gráficos?}

Analisando os resultados obtidos em relação aos três grupos da EJA que construíram gráficos (pelo menos um dos dois gráficos propostos), observamos que apenas 30\% dos estudantes do G1 construíram gráficos, enquanto 90\% do G2 e todos os alunos do G3 construíram gráficos.

Considerando os resultados acima, foi realizada uma análise de variância (ANOVA), tendo como variável independente o Grupo (escolarização) e variável dependente, o total de gráficos construídos. Os resultados indicaram efeitos da escolarização $(\mathrm{F}=12,522, \mathrm{p}<0,000)$. Sendo assim, podemos considerar que a escolarização foi um aspecto positivo nas atividades de construção de gráficos. Ao mesmo tempo, reforça a importância de que a atividade de construção de 
gráficos seja algo contínuo na escola, trabalhada desde os anos iniciais, e que não seja priorizada apenas nos anos finais do Ensino Fundamental e Ensino Médio.

A partir dos gráficos construídos pelos estudantes da EJA foram observadas várias dificuldades. De modo geral, observamos que aspectos importantes à compreensão de um gráfico foram pouco incluídos no momento da construção, como o título, a descrição das categorias ou variáveis do eixo das abscissas e a nomeação dos eixos. Dificuldades com a construção proporcional da escala no eixo das ordenadas também foram observadas, como pode ser visto na Tabela 2.

Tabela 2 - Percentual de elementos incluídos nas atividades de construção

\begin{tabular}{l|c|c|c}
\hline \multirow{2}{*}{ Elementos } & \multicolumn{2}{|c|}{ Atividades } & Total \\
\cline { 2 - 4 } & Construção1 & Construção2 & 3,03 \\
\hline Título & 6,25 & & 6,06 \\
\hline Nomeação dos eixos & 6,25 & 5,9 & 69,7 \\
\hline $\begin{array}{l}\text { Descrição das variáveis do } \\
\text { eixo x }\end{array}$ & 93,75 & 47 & 12,1 \\
\hline Proporcionalidade da escala & 6,25 & 17,7 & \\
\hline
\end{tabular}

Em muitos gráficos apenas foram introduzidos valores numéricos (as variáveis dos eixos foram representadas ou de apenas um dos eixos). A supressão de títulos e a falta de descrição de variáveis foram alguns dos problemas que permearam o momento da construção, dificultando a identificação do que estava sendo tratado. Gitirana, Guerra e Selva (2005) observaram as mesmas dificuldades quando, professoras do Ensino Fundamental, foram solicitadas a construírem gráficos. Esse é um ponto crucial, pois se o gráfico por si só deve se caracterizar como uma representação rápida e clara de informações que se quer transmitir (TOLEDO; OVALLE, 1985), poucos foram os gráficos construídos que atingiram tais objetivos.

Duas dificuldades foram observadas no momento da construção da escala: a proporcionalidade e a linha de base. Apenas $12,1 \%$ dos gráficos construídos apresentaram a escala proporcionalmente adequada. No exemplo da Figura 1, a seguir, podemos observar a dificuldade do estudante em estabelecer uma relação proporcional entre os valores da escala. Podemos observar que ele tentou representar os valores apenas de modo aproximado, note que entre 46 e 93 representado na escala não há uma distância proporcional, nem por agrupamento, nem em milímetros. 


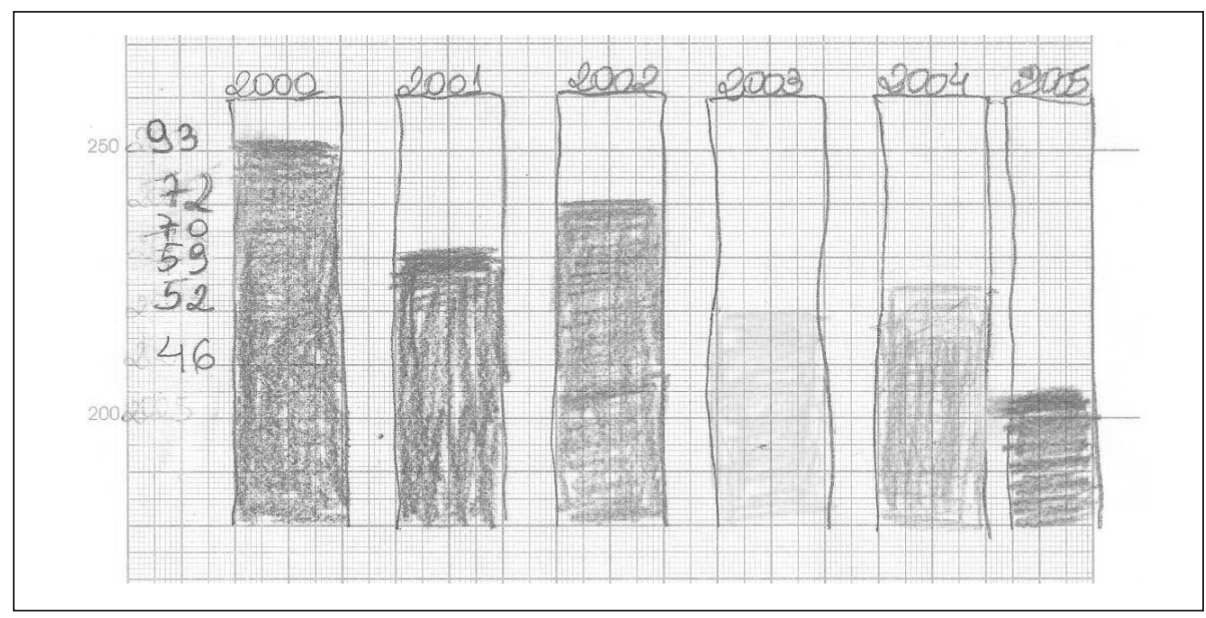

Figura 1 - Gráfico de barras construído por um estudante do grupo G2. Atividade C-1

Para Ainley (2000) o uso de escalas é um marcador das dificuldades enfrentadas pelos estudantes. Entretanto, Guimarães (2002) observou que crianças pequenas foram capazes de estabelecer uma escala correta para representar os dados, apesar de não saberem como utilizá-la. Neste sentido, podemos afirmar que o papel do professor é extremamente importante para auxiliar os estudantes a refletirem sobre a construção de uma escala precisa, chamando a atenção para aspectos como a linha de base, o zero como marco inicial, na decisão de que tipo de escala deve ser adotada e como definir intervalos proporcionais entre os valores da escala.

Para analisar as possíveis relações entre construir e interpretar, observamos o desempenho obtido pelos estudantes em relação ao desempenho nas atividades realizadas, ou seja, observamos o desempenho desses participantes ao longo das cinco atividades propostas (as três de interpretação e as duas de construção). Analisamos cada estudante que construiu adequadamente ao menos um gráfico na atividade de construção (quando houve adequação da escala, da escolha do tipo de gráfico, inclusão de todos os dados necessários à compreensão do gráfico construído etc.) e comparamos com o seu desempenho nas atividades de interpretação. Da mesma forma, observamos os estudantes que responderam corretamente todas as atividades de interpretação e comparamos com seu desempenho nas atividades de construção.

Considerando a análise realizada, podemos sugerir pouca relação entre os desempenhos dos alunos que conseguiram realizar com sucesso as atividades 
propostas de interpretação e os seus respectivos desempenhos em construção. Bons resultados em interpretação não pareceram garantir a construção adequada de um gráfico. Baixos resultados de construção corresponderam, em alguns estudantes, principalmente do grupo G1, a resultados bons em interpretação. Quando consideramos os casos de êxito em construção, vale citar um único estudante que obteve excelente resultado em construção e que também demonstrou um bom resultado em interpretação. Como temos apenas um caso de sucesso na atividade de construção, não podemos tirar conclusões mais gerais, entretanto, consideramos que outros estudos deveriam se debruçar sobre a mesma questão.

\section{Conclusões}

Inicialmente, ressaltamos a importância de se investigar o desempenho de alunos da EJA, modalidade com poucas pesquisas na área de Matemática, nos cenários nacional e internacional. Como sabemos, os alunos da EJA apresentam características peculiares decorrentes de inúmeros processos históricos, políticos, educacionais e sociais, como a exclusão do sistema regular de ensino e a constituição de singularidades socioculturais.

Os resultados obtidos neste estudo mostraram que a influência significativa da escolarização foi observada apenas nas atividades de construção de gráficos, ainda que muitas dificuldades tenham sido observadas, mesmo entre os estudantes do Ensino Médio. Quanto à interpretação de gráficos, nossos resultados indicaram que não houve diferença significativa no desempenho dos estudantes dos três segmentos de ensino ao interpretarem os gráficos propostos.

Considerando, ainda, as atividades de interpretação e de construção, é interessante notar que, de modo geral, os estudantes da EJA, ignorando-se as etapas de escolaridade, parecem ter mais familiaridade e facilidade em interpretar gráficos do que em construir. Nas atividades de construção, além de observarmos mais gráficos construídos em função da escolarização, também observamos nos gráficos construídos muitos elementos ausentes e dificuldades conceituais, como no caso da construção de escalas.

Devemos sugerir que o trabalho com gráficos seja repensado em sala de aula, estabelecendo-se relações com conhecimentos prévios dos jovens e adultos, mas proporcionando a análise da especificidade da representação gráfica. Também, é preciso estimular a permanência dos alunos na escola, através de aulas contextualizadas e atrativas. Trabalhar com gráficos pode contribuir nessa 
direção, pois vários temas interessantes e atuais podem ser discutidos em sala de aula, estimulando a construção de uma matemática que faça sentido para os

estudantes. É preciso valorizar os conhecimentos prévios e reconhecê-los como ponto de partida para novas aprendizagens (SILVA, 2006; GOMES, 2007).

As questões discutidas no presente estudo sugerem a importância de se realizar outras pesquisas, com um quantitativo maior de gráficos representados por diferentes tipos de variáveis e temáticas, com o objetivo de investigar quais são os efeitos no desempenho de estudantes em função desses aspectos e quais são os conhecimentos mobilizados quando os alunos são solicitados a interpretarem e construírem representações gráficas.

Segundo Vergnaud (1986), para compreender a apropriação dos conhecimentos se faz necessário estudar conjuntos bastante vastos de situações. Esperamos, desse modo, avançar com algumas questões relacionadas à compreensão dos conhecimentos estatísticos e matemáticos presentes, tanto em relação às situações-problema envolvidas em atividades de interpretação de gráficos, quanto à proposição de atividades de construção. Acreditamos, assim, que novas perspectivas para o ensino e aprendizagem de conteúdos matemáticos referentes ao bloco Tratamento da Informação possam ser pensadas, em especial para os jovens e adultos que iniciam ou retomam o processo de escolarização formal.

\section{Referências}

AINLEY, J. Exploring the transparency of graphs and graphing. In: CONFERENCE OF THE INTERNATIONAL GROUPFOR THE PSYCHOLOGY OF MATHEMATICS EDUCATION, 24 ${ }^{\text {th }}, 2000$, Hiroshima. Proceedings... Hiroshima: PME, 2000, V.2, p. 9-16.

BRASIL. Secretaria de Educação Fundamental. Parâmetros curriculares nacionais: matemática. Brasília: MEC/SEF, 1998.

GARFIELD, J. The Challenge of Developing Statistical Reasoning. Journal of Statistics Education, Minnesota, v.10, n.3, p.1-12, Nov. 2002.

GITIRANA, J. M. de A. C.; GUERRA, S. E. M. S.; SELVA, A. C. V. Professores construindo e interpretando gráficos de barras: um estudo exploratório. 2005. $25 f$. Trabalho de Conclusão de Curso (Graduação em Pedagogia) - Universidade Federal de Pernambuco, Recife, 2005. 
GOMES, M. J. Profissionais fazendo matemática: o conhecimento de números decimais de alunos pedreiros e marceneiros da Educação de Jovens e Adultos. 2007. 207f. Dissertação (Mestrado em Educação) - Centro de Educação, Universidade Federal de Pernambuco, Recife, 2007.

GUIMARÃES, G. L., GITIRANA, V., ROAZZI, A. Interpretando e construindo gráficos. REUNIÃO ANUALDAANPED, 24., 2001, Caxambu. Anais... Caxambu: ANPED, 2001. p.1-19. Disponível em: <www.anped.org.br/reunioes/24/tp1.htm\#gt19> Acesso em: 02 abr. 2013.

GUIMARÃES, G. L. Interpretando e construindo gráficos de barras. 2002. 260f. Tese (Doutorado em Psicologia Cognitiva) - Universidade Federal de Pernambuco, Recife, 2002.

LOPES, C. A. E. Literacia estatística e INAF 2002. In: FONSECA, M. da C.F.R. (Org.). Letramento no Brasil: habilidades matemáticas. São Paulo: Global, 2004. p.187-197.

LOPES, C. A. E. A implementação curricular da Estatística e da Probabilidade na Educação Básica. SEMINÁRIO INTERNACIONAL DE PESQUISA EM EDUCAÇÃO MATEMÁTICA, 4., 2009, Brasília. Anais... Brasília: Universidade Católica de Brasília, 2009. p.01-12. CD-ROM.

NUNES et al. Introdução à Educação Matemática. São Paulo: PROEM, 2002.

LOPES, C. A. E.; CARVALHO, C. Literacia Estatística na educação básica. In: LOPES, C. A. E.; NACARATO, A. M. (Orgs.) Escritas e leituras na Educação Matemática. Belo Horizonte: Autêntica, 2005.

OLIVEIRA, M. K. de. Jovens e adultos como sujeitos de conhecimento e aprendizagem. Revista Brasileira de Educação, São Paulo, n.12, p. 59-73, set/out/nov/ dez. 1999.

PAGAN, A. et al. A leitura e interpretação de gráficos e tabelas no Ensino Fundamental e Médio. SIMPÓSIO INTERNACIONAL DE PESQUISA EM EDUCAÇÃO MATEMÁTICA SIPEMAT, 2., 2008, Recife. Anais... Recife: Universidade Federal Rural de Pernambuco, 2008. p.01-10. CD-ROM ISBN: 978-8587459-81-7.

SELVA, A. C. V. Gráficos de barras materiais manipulativos: analisando dificuldades e contribuições de diferentes representações no desenvolvimento da conceitualização matemática em crianças de seis a oito anos. 2003. 226f. Tese (Doutorado em Psicologia Cognitiva) - Universidade Federal de Pernambuco, Recife, 2003. 
SILVA, V. L. da. Números decimais: no que saberes de adultos diferem dos de crianças? 2006. 202f. Dissertação (Mestrado em Educação) - Centro de Educação, Universidade Federal de Pernambuco, Recife, 2006.

TOLEDO, G. L.; OVALLE, I. I. Estatística Básica. 2. ed. São Paulo: Atlas, 1985.

VERGNAUD, G. Psicologia do desenvolvimento cognitivo e didáctica das matemáticas. Um exemplo: as estruturas aditivas. Análise Psicológica, Lisboa, v.1, n.5, p.75-90, 1986.

Submetido em Abril de 2012. Aprovado em Junho de 2012. 